\title{
Single Lung Retransplantation for Graft Infarction due to Herniation of Heart
}

\author{
Prashant N. Mohite ${ }^{1}$ Anton Sabashnikov ${ }^{1}$ Praveen Rao ${ }^{2} \quad$ Bartlomiej Zych ${ }^{1}$ André Simon ${ }^{1}$
}

1 Department of Cardiothoracic Transplantation and Mechanical Circulatory Support, Royal Brompton \& Harefield NHS Foundation Trust, London, Middlesex, United Kingdom

2 Department of Radiology, Royal Brompton \& Harefield NHS

Foundation Trust, London, Middlesex, United Kingdom

\begin{abstract}
Address for correspondence Anton Sabashnikov, MD, Department of Cardiothoracic Transplantation and Mechanical Circulatory Support, Royal Brompton \& Harefield NHS Foundation Trust, Harefield Hospital Hill End Road Harefield, London, Middlesex UB9 6JH, United Kingdom (e-mail: a.sabashnikov@rbht.nhs.uk).
\end{abstract}

Thorac Cardiovasc Surg Rep 2013;2:40-42.
Abstract
Keywords
- primary graft infarction
- lobar lung transplantation
- lung transplantation

A young woman with terminal respiratory failure due to idiopathic pulmonary hypertension underwent bilateral lung transplantation. The postoperative course was complicated by herniation of the heart through over the cut pericardial edge on left side leading to left-sided graft infarction requiring pneumonectomy. Unable to wean off mechanical ventilation, patient required lobar transplantation on the left side.

\section{Introduction}

Lung transplantation has now been accepted as routine treatment for end stage respiratory failure due to various etiologies. ${ }^{1}$ Graft infarction after lung transplantation is a rare but known complication requiring graft excision. We present a case in which herniation of heart through pericardial rent led to graft infarction.

\section{Case Report}

A 28-year-old housewife presented 5 years ago, with shortness of breath and decreased exercise tolerance. Over the years, her breathlessness increased in severity and she started complaining of orthopnea and pedal edema. At that time, her chest X-ray showed cardiomegaly, her echocardiography report demonstrated moderate to severe systolic dysfunction of the right ventricle, and her computed tomogram showed a dilated main pulmonary artery $(3.5 \mathrm{~cm})$. A subsequent cardiac catheterization revealed a mean pulmonary artery pressure of $59 \mathrm{~mm} \mathrm{Hg}$ and a pulmonary vascular resistance of 16 Wood units. She was started on bosentan, furosemide, and overnight oxygen at home. She was referred to our unit and after assessment, was accepted on waiting list for lung transplan- tation. After accepting a suitable donor offer, she underwent double lung transplantation via Clamshell incision under cardiopulmonary bypass ( $\mathrm{CPB}$ ). After weaning off $\mathrm{CPB}$, the patient developed recurring ventricular fibrillation with critical hemodynamic instability, necessitating central extracorporal membrane oxygenator (ECMO) system, which was implanted utilizing right atrial and aortic cannulae and was transferred to the intensive care unit (ICU) under stable conditions. Postoperatively, the patient was maintained on moderate inotropic support and nitric oxide. On second postoperative day, due to persistent haziness over the left upper lobe on chest X-ray and continuing left bronchial edema on bronchoscopy, a computed tomographic (CT) scan was performed. It showed displacement of heart into the left hemithorax, congestion in the left upper lobe with poor return from the left pulmonary veins (-Fig. 1). The patient was taken to the theater and reexplored, where the heart was found herniating over the pericardial edge into left hemithorax, strangulating the left pulmonary artery and the veins. The heart was replaced into pericardial cavity and stay suture was taken for fixing the pericardium to the left chest wall.

Serial bronchoscopies over the next 5 days showed increasing congestion and edema in the left upper lobe bronchus, received

May 3, 2013

accepted after revision

August 4, 2013

published online

September 26, 2013
DOI http://dx.doi.org/

10.1055/s-0033-1356750.

ISSN 2194-7635. (c) 2013 Georg Thieme Verlag KG
Stuttgart · New York

License terms

$\Theta(1) \Theta \Theta$ 


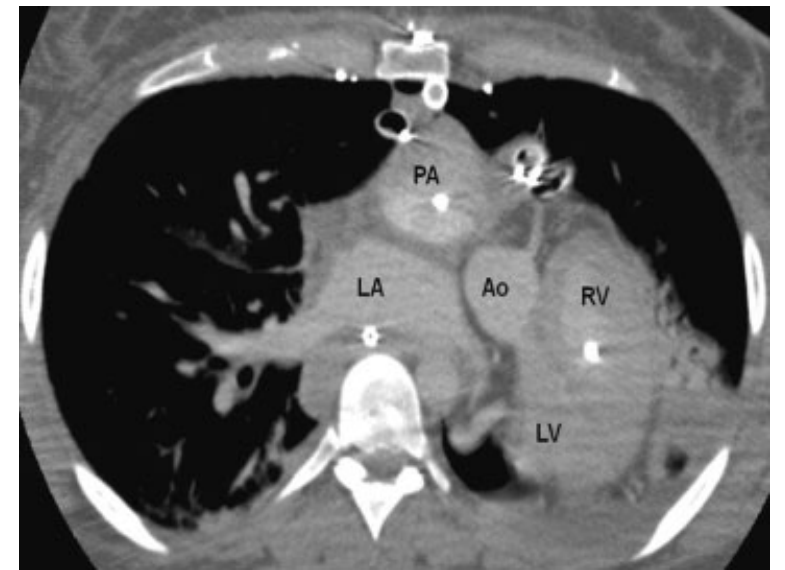

Fig. 1 Computed tomographic scan showing herniation of heart in left hemothorax.

while X-ray and CT scan showed collapsed left upper lobe with obstruction in left pulmonary artery (-Fig. 2). The chest was reexplored for the second time. During surgery, a complete necrosis of the left upper lobe was detected, necessitating a resection. Unfortunately, after upper lobectomy, it became apparent that the majority of lower lobe also showed signs of infarction and necrosis. At this point, a full left pneumonectomy was performed and the bronchial stump was closed with interrupted sutures and protected with a mobilized pericardial flap. The left hemithorax was filled with three breast implants $(1 \times 500 \mathrm{~mL}$ and $2 \times 250 \mathrm{~mL})$. After removal of the graft, the patient remained hemodynamically stable with adequate ventilation parameters. The ECMO was weaned and the patient was returned to the ICU. The histopathological examination of the graft demonstrated diffused hemorrhagic infarct with extensive thrombotic material in the left pulmonary artery and its branches. The subsequent postoperative course was characterized by slow weaning from the ventilator necessitating a tracheostomy. During this time, it became apparent, that it was unlikely that the patient would be able to wean off mechanical respiratory support. Thus, after extensive interdisciplinary discussions, she was reassessed for a left-sided single

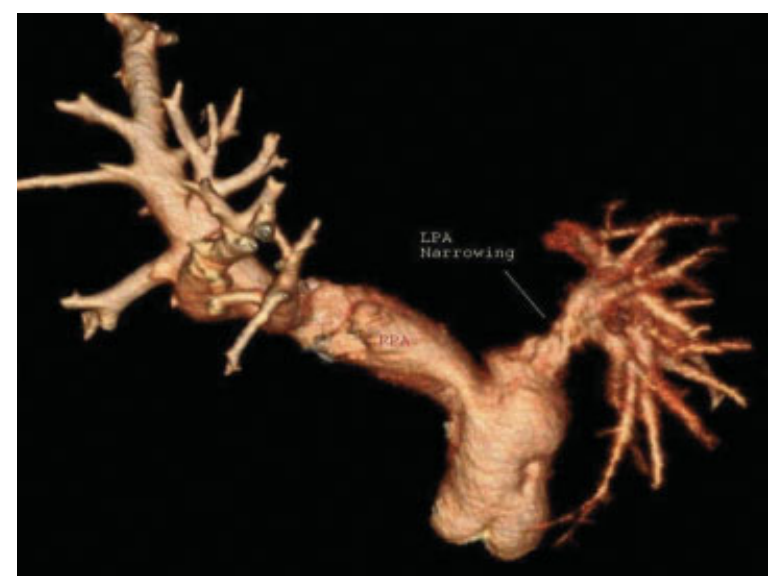

Fig. 2 Three-dimensional reconstruction of pulmonary artery showing obstruction of left pulmonary artery. lung retransplantation and was subsequently placed on the waiting list. As a complicating factor, the patient developed anti-human leukocyte antigen (anti-HLA) antibodies with 35\% panel reactive antibodies. As a consequence, the perioperative protocol included plasmapheresis for immunoadsorption and tailored immunosuppression.

After spending 101 days on waiting list, a suitable donor was found, albeit with a significant size mismatch. Due to the long waiting time, the clinical status of the patient and the HLA match, the donor was accepted and the patient underwent left sided retransplantation with the donor left upper lobe. The breast implants, which helped avoiding obliteration of left hemothorax, were removed at the time of surgery. Postoperatively, the patient made rapid progress and was discharged to ward within 4 days and to home within a month of surgery. Over a year of follow up, the patient remains stable, active, without oxygen therapy, with forced expiratory volume in 1 second (FEV1) near $60 \%$ of the predicted.

\section{Discussion}

Infarction of the pulmonary graft after transplantation is a rare complication. In most cases, it is caused by a pulmonary artery embolism or stenosis of arterial or venous anastomosis. Two cases of pulmonary embolism leading to pulmonary infarction after lung transplantation causing significant morbidity are reported. ${ }^{2}$ Isolated pulmonary vein obstruction can be responsible for total or lobar lung infarction. ${ }^{3,4}$ It could be due to narrow anastomosis or faulty surgical technique. In our case, herniation of heart over the pericardial edge strangulated the left pulmonary artery. This could be because of excess opening of pericardial cavity for CPB and ECMO cannulae. Also, dilated and hypertrophied heart due to idiopathic pulmonary hypertension (IPH) could have jumped over the pericardial edge. Change in the anatomical configuration due to central ECMO cannulae as a cause of herniation could not be ruled out.

The requirement to open the pericardium in lung transplantation (LTx) is minimized with the advent of off-pump surgery and approach to individual lungs through bilateral anterolateral minithoracotomies. In cases of idiopathic pulmonary fibrosis and pulmonary hypertension, one can choose to perform LTx under CPB via femoral cannulation or peripheral ECMO, which could be left in for few days after surgery.

In graft infarction, the bronchoscopic findings may include discoloration of the bronchial tree and localized edema, and intrabronchial fluid discharge. The chest X-ray may show consolidation, atelectasis, increased pulmonary vascular markings or increased hilar size. A CT scan with contrast may provide valuable information about obstruction in pulmonary arteries and pulmonary venous return. Pulmonary angiography can demonstrate obstruction or thrombus at arterial or venous anastomotic site. ${ }^{5}$ These clinical and radiological features can easily be mistaken as reperfusion injury, consolidation of the lung, or left-right side myocardial pump mismatch resulting in temporary postoperative increased pulmonary artery pressures despite a normal vascular resistance. By the time the diagnosis of graft infarction is 
confirmed, there is irreversible damage to the lung parenchyma, which happened in the present case as well. The treatment options are extremely limited at this stage and usually associated with significant morbidity. For instance, Malden et al report graft infarction due to isolated pulmonary vein obstruction following single lung transplantation on left side requiring retransplantation within 10 days. ${ }^{3}$ Pulmonary segmental venous infarction after living-donor lobar lung transplantation was treated conservatively as it involved only one segment. ${ }^{4}$ Left inferior lobectomy was performed in a case of pulmonary artery embolism. ${ }^{6}$ Catheter lysis was tried with short-term improvement in graft function. ${ }^{7}$ In the present case, the cause of graft infarction was pulmonary venous strangulation due to herniation of the heart into the left thorax. On X-ray and CT scan the changes were limited to upper lobe and could not be differentiated from consolidation. By the time of reexploration, both upper and lower lobes were involved and the graft was not salvageable. To our knowledge, this is the first report of this kind in which herniation of heart was the cause of graft infarction and the patient was managed on transplanted single lung for more than 3 months before undergoing a retransplantation.

\section{Conclusions}

Herniation of heart over the pericardial edge following lung transplantation is rare; however, it may cause graft infarction due to strangulation of vessels. Pericardium should be cut carefully and only over the aorta and right atrium, if CPB or ECMO support is required. Extra care should be taken in case of dilated and big hearts, which are often seen in idiopathic and secondary pulmonary hypertension. One should suspect pulmonary infarction in cases of persistent dubious radiological findings, perform pulmonary angiography to rule out mechanical obstruction in pulmonary artery and veins, and in suspected cases reexploration should not be delayed.

\section{References}

1 Trulock EP, Christie JD, Edwards LB, et al. Registry of the International Society for Heart and Lung Transplantation: twenty-fourth official adult lung and heart-lung transplantation report-2007. J Heart Lung Transplant 2007;26(8):782-795

2 Krivokuca I, van de Graaf EA, van Kessel DA, van den Bosch JM, Grutters JC, Kwakkel-van Erp JM. Pulmonary embolism and pulmonary infarction after lung transplantation. Clin Appl Thromb Hemost 2011;17(4):421-424

3 Malden ES, Kaiser LR, Gutierrez FR. Pulmonary vein obstruction following single lung transplantation. Chest 1992;102(2):645-647

4 Choong CK, Hu DZ, Huddleston CB. Pulmonary segmental venous infarction after living-donor lobar lung transplantation. J Thorac Cardiovasc Surg 2005;130(3):919-921

5 Williamson WA, Tronic BS, Levitan N, Webb-Johnson DC, Shahian DM, Ellis FH Jr. Pulmonary venous infarction. Chest 1992;102(3): 937-940

6 Zegdi R, Dürrleman N, Achouh P, et al. Unilateral pulmonary edema after pulmonary embolism in a bilateral lung transplant patient. Ann Thorac Surg 2007;84(6):2086-2088

7 Suhling H, Westerkamp V, Dinh QT, et al. Catheter fragmentation and local lysis in two lung transplant patients with pulmonary embolism. Am J Transplant 2010;10(11):2553-2557 